\title{
Infectious Mononucleosis with Massive Eosinophils and Community-Acquired Pneumonia Due to Three Atypical Pathogens Infections in An Adult: A Case Report
}

\author{
Lifang Peng and Shaohui Tang* \\ Department of Gastroenterology, The First Affiliated Hospital, Jinan University, China
}

*Corresponding author: Shaohui Tang, Department of Gastroenterology, The First Affiliated Hospital, Guangzhou, Guangdong, China

\section{ARTICLE INFO}

Received: 崫 July 16, 2019

Published: 慧 July 31, 2019

Citation: Lifang Peng, Shaohui Tang. Infectious Mononucleosis with Massive Eosinophils and Community-Acquired Pneumonia Due to Three Atypical Pathogens Infections in An Adult: A Case Report. Biomed J Sci \& Tech Res 20(2)2019. BJSTR. MS.ID.003412.

\section{ABSTRACT}

Infectious mononucleos is an Epstein-Barr virus (EBV)-induced disease prevalent during preadolescence, predominantly has highly elevated lymphocytosis with a more than $10 \%$ share of atypical lymphocytes. We presented a case of an adult who developed concurrent Epstein-Barr virus and three atypical pathogens infections. And in this case, eosinophils were increased significantly while lymphocytes were normal and the test of atypical lymphocytes is negative, which are very rare in EBV Infectious mononucleosis.

Keywords: Infectious Mononucleosis; Epstein-Barr Virus; Massive Eosinophils; Co-Infection; Atypical Pneumonia; Mycoplasma Pneumoniae; Chlamydia Pneumoniae; Legionella Pneumophila

Abbreviations: CT: Computed Tomography; EBV; Epstein-Barr virus; LP: legionella Pneumophila; VCA: Viral Capsid Antigen; MP: Mycoplasma Pneumoniae; CP: Chlamydia Pneumoniae

\section{Case Report}

A 28-year-old man had a one-month history of fever, mild cough, and abdominal distension. He was started on cephalosporin antibiotic for the suspicion of upper respiratory infections but returned to our hospital one week later with continued fevers. On admission the patient had a high fever of $39.2^{\circ} \mathrm{C}$. Physical examination revealed enlarged pharyngeal tonsils, bilateral inguinal lymphadenopathy and slight epigastric tenderness. Cephalosporin and antipyretic were ineffective for improving his clinical symptoms. His body temperature ranged from $38.2^{\circ} \mathrm{C}$ to $39.2^{\circ} \mathrm{C}$. Laboratory analysis showed high leucocytosis $\left(22.93 \times 10^{9} / \mathrm{L}\right.$ with $73.8 \%$ eosinophils and no atypical lymphocytes) (Figure 1), mildly elevated liver enzymes (alanine transaminase, $83 \mathrm{U} / \mathrm{l}$ ). Laboratory testing was negative for mycobacterium tuberculosis, viral hepatitis A, B, C, D and E, influenza, dengue, leptospira, epidemic hemorrhagic fever, cytomegalovirus, Herpes simplex virus, typhoid fever, Liver fluke, autoimmune antibody, parasites, blood culture and bone marrow. Ultrasound showed left neck, bilateral inguinal, liver and peripancreatic region lymphadenopathy. Chest and abdominal computed tomography (CT) scan revealed left upper-lobe infiltrates, multiple small areas of low density in liver parenchyma (Figures 2 \& 3). Liver biopsies identified acute inflammation of huge amounts of eosinophils (Figure 4). 


\section{患者名字: 刘子为 \\ 性别: 男 \\ 年 离: 28 岁 \\ 送检时间: 2017/11/27 9:47:20 \\ 备注：EOS结果已镜下佘查。

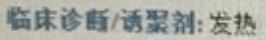

\section{报告单信息}

麻䣦号: 1906780000311972

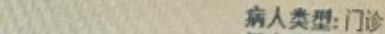

本号: 标本类型: 全血

東校时间: 2017/1127 102020

\begin{tabular}{|c|c|}
\hline 监床意义 & 项目名称 \\
\hline 9 & 白细胞计数WBC \\
\hline 98 & 嗜中性粒细胞百分比 \\
\hline \% & 淋巴细胞百分比 \\
\hline 웡 & 单核细胞百分比 \\
\hline 啧 & 譛酸性细胞百分比 \\
\hline 9 & 譛碱性细胞百分比 \\
\hline 98 & 㖺中性粒细胞绝对值 \\
\hline 9 & 淋巴细胞绝对值 \\
\hline ๑ & 单核细胞绝对值 \\
\hline 원 & 啨酸性细胞绝对值 \\
\hline 果 & 㖺瑊性细胸绝对值 \\
\hline 92 & 红细胞计数RBC \\
\hline 包 & 血红蛋白HGB \\
\hline
\end{tabular}

结果

22.93

10.8

11.7

3.1

73.8

0.6

2.48

2.69

0.71

16.92

0.13

5.07

146

Figure 1: Blood test on November 27, 2017 (high leucocytosis $\left(22.93 \times 10^{9} / \mathrm{L}\right)$ with $73.8 \%$ eosinophils).

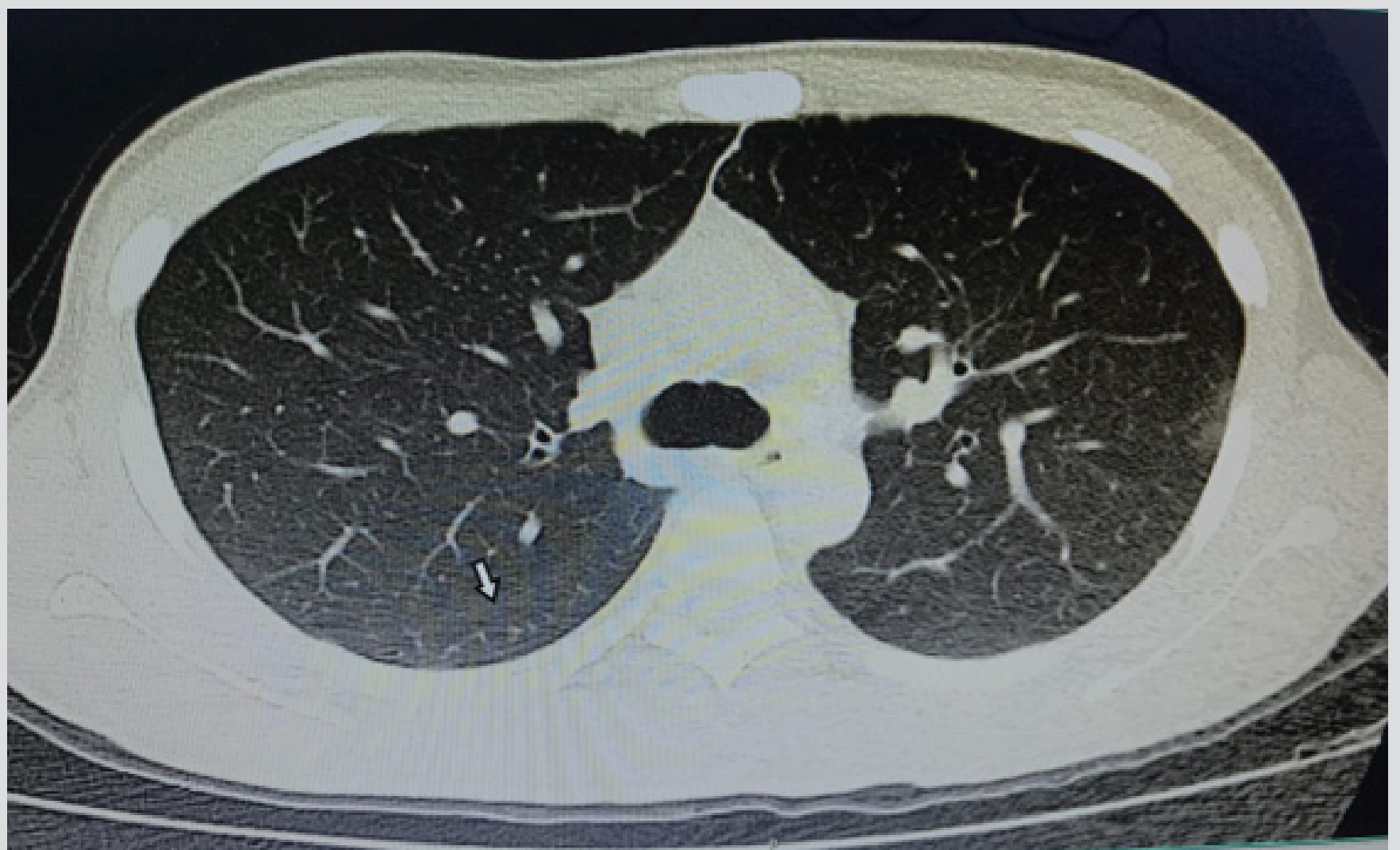

Figure 2: Chest CT enhancement scan (left upper-lobe infiltrates). 


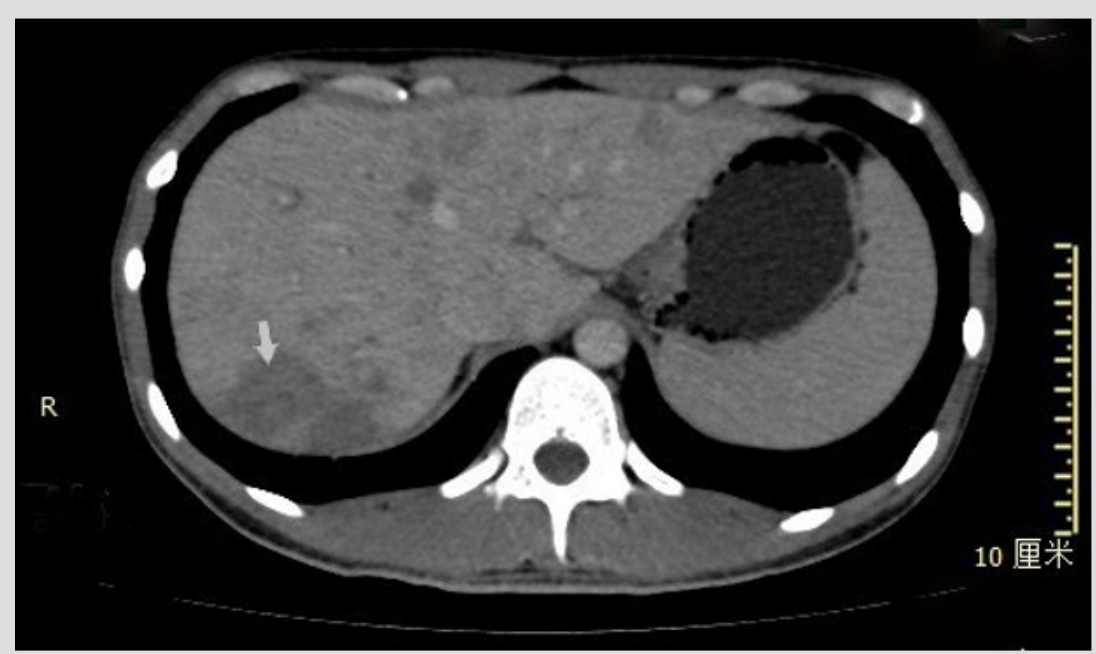

Figure 3: Enhanced CT of abdomen (multiple small areas of low density in liver parenchyma).

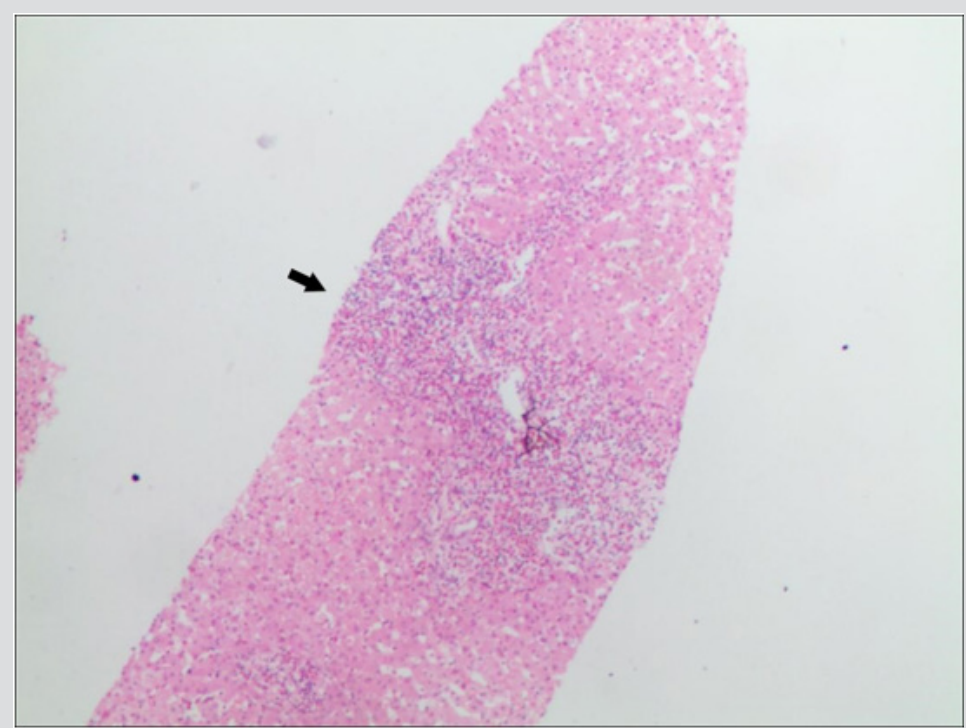

Figure 4: liver biopsy (acute inflammation of huge amounts of eosinophils).

These findings preliminarily excluded rheumatic diseases, parasitic diseases, the common virus infection, hematologically related diseases and tumors. To determine the cause of fever, interrelated tests of other unusual pathogens were examined further. IgM antibodies to Epstein-Barr virus (EBV) viral capsid antigen (VCA), Mycoplasma pneumoniae (MP), Chlamydia pneumoniae (CP) and legionella pneumophila (LP) were positive, suggesting multiple infection. EBV DNA was positive $\left(5.4 \times 10^{5}\right.$ copy $/ \mathrm{ml}$ ) while heterophil agglutination test was negative. Based on these clinical features and serological studies, the patient was diagnosed with infectious mononucleosis (IM) and communityacquired pneumonia caused by three atypical pathogens (MP, CP, LP). Therefore, the patient was started on anti-infective therapy with ganciclovir, erythromycin and ciprofloxacin. After four days his temperature dropped to normal and then he was discharged and monitored in the outpatient clinic. At the five-week follow-up, his white blood cells and eosinophil were completely normal.

\section{Discussion}

Infection mononucleosis (IM) is an acute infectious disease which is caused by EBV, spreads through droplet and usually occurs during preadolescence. Clinical presentations are composed of nonspecific symptoms, including fever, sore throat, lymphadenopathy, splenomegaly and hepatomegaly [1]. High lymphocytosis with atypical lymphocytes is highly suggestive of EBV IM, and elevated IgM antibodies and EBV DNA is the most accurate method to confirm the diagnosis. Although IM is a self-limiting illness, it can involve many important organs and systems and vary greatly in symptoms, which lead to its high misdiagnosis rate. Antibiotics were ineffective and this was the reason why fever had been continued until an antiviral drug was given. Pneumonia caused by atypical pathogens, including MP, CP and LP can be called Atypical pneumonia [2]. It can be confirmed by symptoms (fever, sore throat, cough, and so on), blood picture (reduced or normal leucocyte), chest radiograph and 
the detection of atypical pathogens specific antibodies (positive IgM antigens).Here, we presented an exceptional case of IM which has the following characteristics:

a) As a 28-year-old healthy adult, the clinical presentation and positive EBV, MP, CP and LP IgM test strongly suggested four pathogens co-infection. No prior case report has described similar scenarios.

b) Though he had typical clinical findings of EBV IM like fever, sore throat and lymphadenopathy, his blood test showed normally lymphocytosis with no atypically lymphocytes and massive eosinophils. It is really rare in IM, only two analogous cases have been reported previously by searching related case reports [3]. Eosinophils in these two cases was only one- eighth of our case. Based on laboratory analysis and CT findings, the patient was excluded differential diagnosis which have high eosinophilia, such as parasitic infection, anaphylaxis, rheumatic diseases, blood system diseases and tumor, and confirmed that massive eosinophilia was the response to mixed infections(EBV, MP, CP and LP) [4]. Failed treatment of common antibiotics and effectivity of antiviral drug and targeted anti-infection drugs were consistent with the diagnosis.

c) This patient was simultaneously infected by four pathogens and diagnosed with four diseases. It is one of its characteristics. We guessed that the patient was infected by one or two types of pathogens first, and the initial infection and decreased immunity and defense function rendered the host more susceptible to co-infection. Atypical pathogens infections, such as EBV, mycoplasma, chlamydial, legionella, dengue and cytomegalovirus usually involve different organs and systems, and therefore the clinical presentations in patients infected with multiple pathogens can vary greatly. In order to avoid misdiagnosis, to obtain early recognition and to give a timely treatment to facilitate a full recovery, it is essential to do comprehensive detection of atypical pathogens earlier for patients presenting with continued fever, atypical symptoms or blood picture and void routine therapy.

\section{References}

1. Xie Zhengde (2007) Clinical features and diagnostic criteria of infectious mononucleosis associated with Epstein-Barr virus infection in children. Appl Clin Pediatr 22(22): 1759-1760.

2. Tong Chuntang, Chen Hangwei (2014) Research development on the diagnosis of atypical pathogens in community acquired pneumonia. Chin J Lung Dis 7(6): 675-678.

3. Wang Tao, Wang Yazhen (1990) Two case reports of Infectious mononucleosis with increasing eosinophils. The Liao Ning Journal of Medicine (3): 149-150.

4. Lin Jie, Wu Yongji (2005) A clinical analysis of 53 cases of hypereosinophilia. Chinese Journal of Practical Medicine 25(11): 10171018.

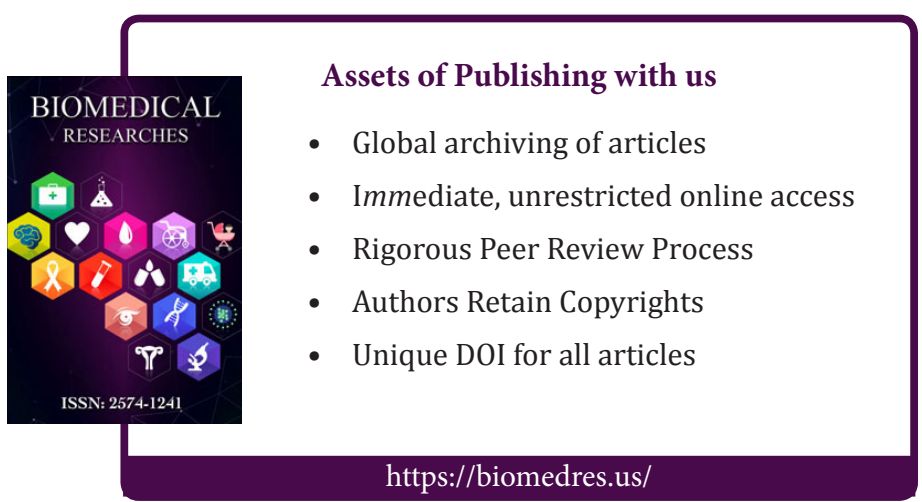

